\title{
Worldsheet of a continuous helicity particle
}

\author{
D. S. Kaparulin, ${ }^{*}$ S. L. Lyakhovich,$^{\dagger}$ and I. A. Retuntsev ${ }^{\ddagger}$ \\ Physics Faculty, Tomsk State University, Tomsk 634050, Russia
}

(Received 13 December 2021; accepted 18 January 2022; published 4 March 2022)

\begin{abstract}
We consider the class of spinning particle theories, whose quantization corresponds to the continuous helicity representation of the Poincare group. The classical trajectories of the particle are shown to lie on the parabolic cylinder with a lightlike axis irrespectively to any specifics of the model. The space-time position of the cylinder is determined by the values of momentum and total angular momentum. The value of helicity determines the focal distance of parabolic cylinder. Assuming that all the world lines lying on one and the same cylinder are connected by gauge transformations, we derive the geometrical equations of motion for the particle. The timelike world paths are shown to be solutions to a single relation involving the invariants of trajectory up to fourth order in derivatives. Geometrical equation of motion is non-Lagrangian, but it admits equivalent variational principle in the extended set of dynamical variables. The lightlike paths are also admissible on the cylinder, but they do not represent the classical trajectories of this spinning particle. The classical trajectories of massless particle (with zero helicity) are shown to lie on hyperplanes, whose space-time position depends on momentum and total angular momentum.
\end{abstract}

DOI: 10.1103/PhysRevD.105.065004

\section{INTRODUCTION}

The spinning particles are studied since the work of Frenkel [1]. For review of results before 1968, we cite the book [2], later studies can be found in [3]. For the more recent research, we refer to $[4,5]$ and references therein. The concept of spinning particle admits generalization in low-dimensional space-times [6,7], and higher dimensions [8-10]. For the constant curvature spaces, we cite [11]. It is believed that the spinning particle models provide the realistic quasiclassical description of the motion of real elementary particles with spin and localized twisted wave packets [12]. For the applications spinning particle concept in high energy and accelerator physics, astrophysics we mention [13-22].

The class of spinning particle models whose quantization corresponds to the irreducible representation of the Poincare group is of interest. The examples are given above. The Kirillov-Konstant-Soureau method [23-25] tells us that the quantization of the model leads to irreducible representation if its classical limit is a dynamical system on the co-orbit of corresponding group. In this

\footnotetext{
*dsc@phys.tsu.ru

†sll@phys.tsu.ru

*retuntsev@phys.tsu.ru
}

Published by the American Physical Society under the terms of the Creative Commons Attribution 4.0 International license. Further distribution of this work must maintain attribution to the author(s) and the published article's title, journal citation, and DOI. Funded by SCOAP ${ }^{3}$. setting, the state of the irreducible particle is determined by the values of momentum $p$ and total angular momentum $J$, being subjected to mass-shell and spin-shell conditions. All the gauge invariant observables are the functions of $p, J$. The action functional is given by the symplectic form on the co-orbit.

The majority of irreducible spinning particle models have one common feature: the generalized coordinates include, besides the particle position, the position in internal space. The configuration space of the model is given by the fiber bundle $\mathbb{R}^{1, d-1} \times \mathbb{S}$, with the internal space $\mathbb{S}$ being typical fiber. The Lagrangian of the model is a function of Lorentz invariant combinations of the generalized coordinates and their derivatives, typically up to the first order. The structure of Lagrangian is selected from the requirement of irreducibility of Poincare group representation. The equations of motion follow from the least action principle, and they inevitably involve internal coordinates. In all these models, the relationship between the representation and classical dynamics is hidden in the structure of Lagrangian. The universal dynamical principle selecting the trajectories of spinning particle has been unknown for a long time.

In the current article, we consider the spinning particle motion using the recently proposed worldsheet concept [26]. In this paper, it has been shown that the classical trajectories of the irreducible spinning particle inevitably lie on the cylindrical (hyper)surface in Minkowski space. The (hyper) surface was termed the worldsheet of spinning particle. The shape of the worldsheet is determined by the representation. The worldsheet position in the space-time is determined by 
the values of the momentum and total angular momentum, being subjected to the mass shell and spin shell conditions. The dynamical equations for the particle classical trajectories follow from the fact that all the world lines that lie on one and the same worldsheet are connected by the gauge transformations. The resulting equations of motion are purely geometrical relations on the particle world line, and they do not involve extra variables.

The geometry of worldsheets of massive spinning particles has been described in the original paper [26]. The world surfaces has been shown to be toroidal cylinders of dimension $[(d+1) / 2]$ with timelike axis (the square brackets determine the integer part of enclosed number). In $d=3,4$, the world surfaces are circular cylinders with timelike axis. The value of momentum determines the direction of symmetry axis, and the value of total angular momentum defines the position of cylinder in Minkowski space. The spinning particle trajectories are general curves on circular cylinders. The geometrical equations of motion in $d=3$ have been derived in [26]. In $d=4$, the same problem has been solved in [27]. An alternative derivation of equations of motion for cylindrical curves has been given in [28]. In all the cases, the differential equations involve invariants of trajectory up to the fourth order in derivatives in a very complicated combination, and they are known only in implicit form. The geometrical equations of motion are non-Lagrangian, but they admit equivalent variational formulation with extra fields. This formulation represents previously known model [6]. The worldsheet concept admits inclusion of interactions with external electromagnetic field [29]. At the interaction level, the spinning particle paths still lie on a two-dimensional hypersurface, but its shape depends on the configuration of external field.

In the articles [30-32], it has been noted that the helices with a lightlike tangent vector and timelike symmetry axis are admissible trajectories for massive particle. In the framework of the worldsheet formalism, these curves represent the cylindrical paths with a lightlike tangent vector. The geometrical equations of motion for lightlike helical paths in $d=3,4$ derived in $[26,27]$. It has been shown that the lightlike helices represent the special class of cylindrical world lines with a reduced gauge symmetry. In so doing, each helix forms the gauge equivalence class of its own. This suggests that the helices can be considered as the one-dimensional spinning particle worldsheets. The construction [30-32] seems to be unique in space-time dimension $d=3,4$ and it applies only for massive particles. We do not know geometrical models of massive particles with lightlike trajectories in higher dimensions. As for massless particles, their positions are known to lie on the hyperplanes $[33,34]$, but the general fact is not proven up to date.

In the present article, we apply the worldsheet concept for the study of dynamics of massless spinning particles with continuous helicity in $d=3$ Minkowski space.
The worldsheets are parabolic cylinders with lightlike symmetry axis. The focal distance of the parabolic cylinder is determined by helicity. The worldsheet position is determined by the values of momentum and total angular momentum. The geometrical meaning of $p$ and $J$ is explicitly identified. Assuming that all the particle trajectories lying on one and the same cylinder are connected by the gauge transformations, we derive the equations of motion for the curves with timelike tangent vector on the worldsheet. We show that the particle paths enjoy a single differential equation involving invariants of trajectory up to the forth order. The equation of motion is nonLagrangian, but it admits equivalent variational formulation with extra dynamical variables, been previously known [6]. The lightlike trajectories are identified with the paths with zero curvature. These paths do not correspond to the trajectories of irreducible particle. The worldsheet of the particle with zero mass and helicity are shown to be hyperplanes. Hence, the trajectories of such particles are proven to be planar curves.

The article is organized as follows. In Sec. II, we describe the geometry of the worldsheet of massless particle with continuous helicity in three-dimensional Minkowski space. In Sec. III A, we derive the equations with hight derivatives for general cylindrical path on the worldsheet. Timelike (Sec. III B) and lightlike (Sec. III C) trajectories are considered. In Sec. IV, we construct Hamiltonian formulation for the model and discuss the correspondence with the previously known model of such a particle. In Sec. V, we discuss the dynamics of massless particles. The conclusion in Sec. VI summarizes the results.

\section{IRREDUCIBILITY CONDITIONS AND WORLDSHEET}

We consider the spinning particle that travels in $3 d$ Minkowski space. The particle position is denoted by $x^{\mu}$, $\mu=0,1,2$, the momentum is $p$ and total angular momentum is $J$. We assume that the quantization of the model corresponds to the irreducible representation of the Poincare group with a continuous helicity. Irreducibility means that the momentum $p$ and total angular momentum $J$ meet the mass-shell and spin-shell conditions,

$$
(p, p)=0, \quad(p, J)=\sigma .
$$

Here, the round brackets denote the scalar product with respect to the Minkowski metric. We use a mostly positive signature of the Minkowski metric $\eta_{\mu \nu}=\operatorname{diag}(-1,1,1)$ throughout the paper. Constant parameter $\sigma$ is helicity. The case $\sigma=0$ corresponds to the massless particle. The values of momentum $p$ and total angular momentum $J$, being subjected to condition (1), determine the state of spinning particle. The space of all the classical spinning particle states is associated with the co-orbit (1) of the Poincare group. 
The vector of spin angular momentum $M$ is determined by the rule

$$
M=J-[x, p],
$$

where the square brackets denote the cross product in $3 d$ space-time. We use the convention

$$
[u, v]=\epsilon_{\mu \nu \rho} u^{\mu} v^{\nu} d x^{\rho}, \quad \epsilon_{012}=1,
$$

where $\epsilon_{\mu \nu \rho}$ is $3 d$ levi-Civita symbol, and $u, v$ are test vectors. In accordance with our definition, the representation for double cross product of three test vectors $u, v, w$ reads

$$
[u,[v, w]]=w(u, v)-v(u, w) .
$$

We note that the last formula is sensitive to the particular choice of the signature of the metric. The article [26] tells us that the spin angular momentum vector $M$ must be normalized in every irreducible spinning particle theory,

$$
(M, M)=\varrho .
$$

The value of $\varrho$ distinguishes representations with one and the same value of helicity $\sigma(1)$.

Conditions (1), (5) have consequences. Combining (5) with the definition of the spin angular momentum (2), we see that the set of particle positions in the state with prescribed values of momentum $p$ and total angular momentum $J$ forms a hypersurface in Minkowski space,

$$
(p, x)^{2}+2(v, x)+a=0 .
$$

Here, $p$ is the particle momentum, and quantities $v, a$ are determined by the total angular momentum $J$ by the following rule:

$$
v=[J, p], \quad a=(J, J)-\varrho .
$$

By definition (7), the momentum $p$ is lightlike, and the vector $v$ is normalized and orthogonal to $p$,

$$
(v, p)=(p, p)=0, \quad(v, v)-\sigma^{2}=0 .
$$

The hypersurface, being defined by Eq. (6), is termed a worldsheet of spinning particle. By construction, it includes all the particle positions with the momentum $p$ and total angular momentum $J$.

The hypersurface, being defined by equation (6), is a parabolic cylinder with lightlike axis (see Fig. 1). The lightlike vector $p$ determines the direction of symmetry axis. The spacelike vector $v$ determines the direction of asymptotes of parabolas, being orthogonal sections of cylinder. The focal distance of parabolic cylinder is

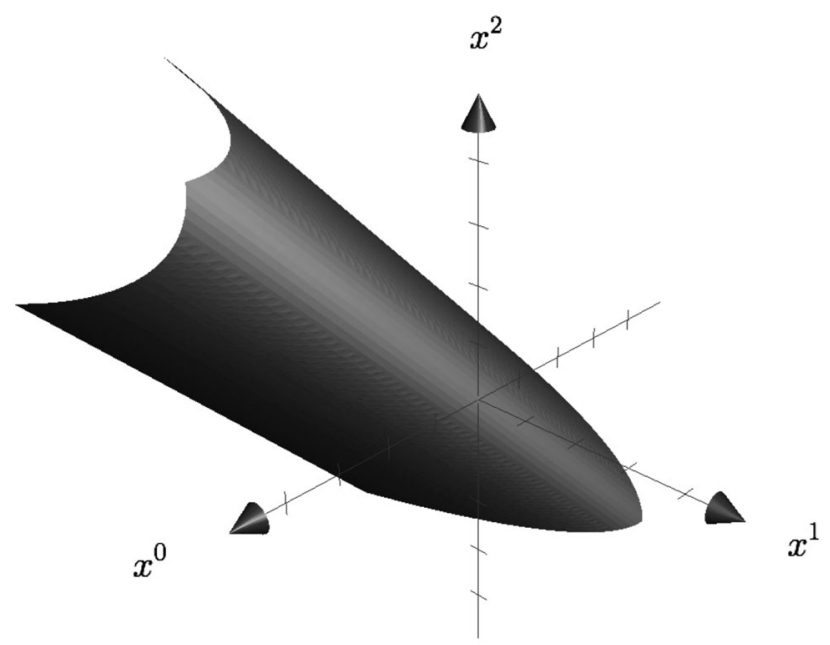

FIG. 1. Worldsheet of continuous helicity particle with $p=(1,0,1), J=(0,0,1), \varrho=1$. The step along the axes equals 1 .

determined the helicity. It equals $\sigma$. The quantity $a$ determines the distance between the vertex of parabolic cylinder and origin. The cylinder parameters determine the total angular momentum $J$ by the rule

$$
J=\frac{a+\varrho}{2 \sigma} v+\sigma e
$$

The relation involves auxiliary lightlike vector $e$ which is orthogonal to $v$ and has a normalized scalar product with $p$,

$$
(e, e)=(e, v)=0, \quad(e, p)=1 .
$$

Equation (6) has consequences. It shows that the classical positions of the particle with continuous helicity lie on a parabolic hypercylinder with lightlike axis in Minkowski space. The position of hypersurface is determined by the momentum and total angular momentum. Formula (7) expresses the values of cylinder parameters $p$, $v, a$ in terms of $p$ and $J$. The relationship between the quantities $p, v, a$, and $p, J$ is a bijection. Formula (9) determines the momentum and total angular momentum of the particle in terms of cylinder parameters. This result means that the co-orbit of the particle with continuous helicity can be parametrized by the set of parabolic cylinders with lightlike axis being the worldsheets. The relationship between the worldsheets and co-orbit points is purely geometrical. The worldsheet is the hypersurface including the all the possible particle positions with given values of $p$ and $J$. The inverse is also true because the space-time position of the worldsheet determines the state of the particle.

Worldsheet concept determines a dynamical principle that governs the particle motion. Equation (6) represents a single restriction imposed onto the particle positions that 
follows from the irreducibility condition of the Poincare group representation. This means that all the points of the worldsheet represent the possible particle positions, while the world paths must be general curves on the worldsheet. Assuming that all the curves that lie on one and the same worldsheet are connected by the gauge transformation, we associate the classical paths of continuous helicity particle with general cylindrical lines on parabolic cylinders with a timelike axis. This reduces the task of description of particle path by differential equations to the problem of classification of cylindrical curves. We elaborate on this problem in the next section.

\section{WORLD PATHS ON WORLDSHEETS}

\section{A. Problem setting}

In the present section, we consider the problem of classification of curves on parabolic cylinders with lightlike axis in Minkowski space. We address three questions: (i) to derive a (system of) differential equations describing the curves on the parabolic cylinder, (ii) to express the parameters of the worldsheet (and, hence, the particle state) in terms of derivatives of world path, and (iii) to identify the gauge symmetries of the model. The problem represents the particular case of more general task of description of the class of curves lying on the set of surfaces. The solution to questions (i), (ii) is well known in the differential geometry of the curves. We cite the textbook [35] for details. The solution to question (iii) is given in [26] for the first time, even though the problem is quite simple in itself.

The description of curves on circular hypercylinder in $3 d$ Euclidean space has been first studied in [36]. The problem has been recently reconsidered in [37,38]. It has been shown that the cylindrical curves are described by a single scalar equation of fourth order. In $3 d$ Minkowski space, the cylindrical curves has been studied in [26]. In $4 d$ Minkowski space, the curves on $2 d$ circular cylinders are classified in [27]. The mentioned above articles use different approaches. In the work [37], the concept of constant separation curves is used. The article [38] assumes that the cylinder is determined by algebraic equation. The last approach is best suited for classification of spinning particle trajectories because the worldsheet is determined by the algebraic equation (6).

In our classification of paths on parabolic cylinders we mostly follow article [37], some additional comments are given in [26]. As the complete solution to the problem involves many technical details, we first explain the general method. The computation details are given in the next subsections. The worldsheet of continuous helicity spinning particle is determined by Eq. (6). The curve $x(\tau)$ lies on the cylinder (6) if the equation of hypersurface is satisfied for all the values of the parameter $\tau$. This implies infinite set of differential consequences,

$$
\frac{d^{k}}{d \tau^{k}}\left((p, x)^{2}+2(v, x)+\left.a\right|_{x=x(\tau)}\right)=0 .
$$

These relations represent an overcomplete system of equations that connects the derivatives of trajectory and cylinder parameters. The relevant information is included into the differential consequences of orders $k=0, \ldots, 4$. It includes five equations for four independent components of $p, v, a$ subjected to (8). Solving these equations with respect to $p, v, a$, we express the parameters of cylinder in terms of parameters of trajectory. This solves the problem (ii). The consistency condition for the system (11) is a differential equation, being satisfied by the cylindrical paths. This equation represents a solution to the problem (i). By construction, it involves the derivatives of world path up to fourth order. The higher-order differential consequences (11) must follow from the lower order ones, so they are not independent. The gauge symmetries of the model are generated by the shifts along the cylindrical surface. So, the gauge generators are associated with the basis vectors in the tangent space to the cylinder. This solves the problem (iii).

The described above procedure has several subtleties. First, the world lines representing the classical trajectories of spinning particles must be causal. The causality condition $\dot{x}^{0}>0$ is imposed to prevent existence of closed world loops, which are considered unphysical. Throughout the article only causal curves are considered. Second, the tangent vector to the Minkowski space curve can be timelike, lightlike, or spacelike. Our analysis shows that the cylindrical curves of different type are not connected by the gauge transformations. So, the classification problems for spacelike, timelike, and lightlike cylindrical curves represent different tasks. In the present work, we consider the timelike and lightlike paths because they automatically meet causality condition. The spacelike curves can be included in general scheme in a similar way, but the causality may be an issue.

Finally, the cylinders can intersect. The intersection line belongs to several cylinders in the set (6), so equations (11) do not have a unique solution with respect to the parameters $p, v, a$. These paths must be excluded because they do not determine a particle state in an unambiguous way. In the case of parabolic cylinders set, the intersection is a line with lightlike tangent vector or noncasual curve. The line appears if the cylinders with one and the same direction of axis are intersect. One line belongs to the infinite number of worldsheets with one and the same direction of symmetry axis. The noncasual curve appears if two cylinders with different directions of symmetry axis intersect. All the mentioned paths are excluded.

In the paper [26], the curves that lie one a unique representative in the class of worldsheets was termed typical. The curves that belong to multiple worldsheets were termed atypical. The classification of timelike and 
lightlike lines on parabolic cylinders presented in Secs. III A and III B considers only typical curves. The atypical curves (including lightlike straight lines) are systematically ignored below.

We use Frenet-Serret moving frame adapted for describing timelike (Sec. III B) and lightlike (Sec. III C) curves [39] to perform our calculations.

\section{B. Timelike world lines on the parabolic cylinder}

Now we can proceed with explicit derivation of the equations of cylindrical path. The differential consequences of (6) up to fourth order have the form

$$
\begin{aligned}
(\dot{x}, n) & =0, \\
(\ddot{x}, n)+(\dot{x}, p)^{2} & =0, \\
(\dddot{x}, n)+3(\dot{x}, p)(\ddot{x}, p) & =0, \\
(\dddot{x}, n)+4(\dot{x}, p)(\dddot{x}, p)+3(\ddot{x}, p)^{2} & =0 .
\end{aligned}
$$

Here, we use a notation,

$$
n=(x, p) p+v \text {. }
$$

The new vector $n$ subjects to the conditions

$$
(p, n)=0, \quad(n, n)=\sigma^{2} .
$$

As we can see from the first equation, vector $n$ defines the normal to tangent space to the worldsheet at the point with coordinate $x$. As far as $n$ is spacelike, the tangent space has the Lorentz signature in each point of the cylinder.

Let us turn to the description of cylindrical curves. Assume that $x(\tau)$ is a timelike world line parametrized by the natural parameter. The velocity vector is normalized,

$$
(\dot{x}, \dot{x})=-1 \text {. }
$$

Throughout the section, the dot denotes the derivative by the natural parameter $\tau$. The Frenet-Serret moving frame, being associated with the timelike curve $x(\tau)$, reads

$$
e_{0}=\dot{x}, \quad e_{1}=\frac{\ddot{x}}{\sqrt{(\ddot{x}, \ddot{x})}}, \quad e_{2}=\frac{[\dot{x}, \ddot{x}]}{\sqrt{(\ddot{x}, \ddot{x})}} .
$$

The basis vectors $e_{a}, a=0,1,2$ of the Frenet-Serret frame are normalized and orthogonal to each other,

$$
\begin{aligned}
-\left(e_{0}, e_{0}\right) & =\left(e_{1}, e_{1}\right)=\left(e_{2}, e_{2}\right)=1, \\
\left(e_{0}, e_{1}\right) & =\left(e_{0}, e_{2}\right)=\left(e_{1}, e_{2}\right)=0 .
\end{aligned}
$$

The vector $e_{0}$ is timelike, and the vectors $e_{a}, a=1,2$ are spacelike. Condition (15) and basis (16) are well defined for each timelike curve, which is not a straight line. This does not restrict generality because no rectilinear paths with timelike tangent vector lie on the parabolic cylinder with the lightlike axis.

The Frenet-Serret formulas for the timelike curve $x(\tau)$ read

$\dot{e}_{0}=\varkappa_{1} e_{1}, \quad \dot{e}_{1}=\varkappa_{1} e_{0}+\varkappa_{2} e_{2}, \quad \dot{e}_{2}=-\varkappa_{2} e_{1}$.

The curvature $\varkappa_{1}$ and torsion $\varkappa_{2}$ of the curve are determined by the rule

$$
\varkappa_{1}=\sqrt{(\ddot{x}, \ddot{x})}, \quad \varkappa_{2}=\frac{(\dot{x}, \ddot{x}, \dddot{x})}{\sqrt{(\ddot{x}, \ddot{x})}}
$$

By construction, the curvature $\varkappa_{1}$ is a positive number, and the torsion $\varkappa_{2}$ is a real quantity. With account for conditions (18), the time derivatives of particle position can be expressed as the linear combinations of the Frenet-Serret basis vectors (16) with the coefficients depending on the curvature and torsion of path and their derivatives,

$$
\begin{aligned}
\dot{x}= & e_{0}, \quad \ddot{x}=\varkappa_{1} e_{1}, \\
\dddot{x}= & \varkappa_{1}^{2} e_{0}+\dot{\varkappa}_{1} e_{1}+\varkappa_{1} \varkappa_{2} e_{2}, \\
\dddot{x}= & 3 \dot{\varkappa}_{1} \varkappa_{1} e_{0}+\left(\ddot{\varkappa}_{1}+\varkappa_{1}^{3}-\varkappa_{1} \varkappa_{2}^{2}\right) e_{1} \\
& +\left(2 \dot{\varkappa}_{1} \varkappa_{2}+\varkappa_{2} \dot{\varkappa}_{2}\right) e_{2} .
\end{aligned}
$$

The representation for $\dddot{x}$ involves the derivative of curvature $\dot{x}_{1}$. The representation for $\dddot{x}$ involves the second derivative of curvature $\ddot{\varkappa}_{1}$, and first derivative of torsion $\dot{\varkappa}_{2}$.

The unknown vectors $p, n$ are determined by the conditions (12) and (14). We seek the solution to (14) in the following form:

$$
\begin{array}{r}
p=\gamma \sqrt{\operatorname{sign}(\sigma) \sigma \varkappa_{1}}\left(e_{0}-\beta e_{1}+\alpha e_{2}\right), \\
n=\operatorname{sign}(\sigma) \sigma\left(\alpha e_{1}+\beta e_{2}\right),
\end{array}
$$

where $\alpha, \beta, \gamma$ are new dimensionless unknowns. The quantities $\alpha, \beta$ are subjected to the condition

$$
\alpha^{2}+\beta^{2}=1
$$

The quantity $\gamma$ is positive because $p^{0}>0$. On substituting representation (21) into (12), we arrive at the following system of algebraic equations for $\alpha, \beta$, and $\gamma$ :

$$
\begin{aligned}
\alpha+\gamma^{2} & =0, \\
\alpha^{2}+\beta^{2}-1 & =0, \\
B \alpha+C \beta+3 \gamma^{2} \beta & =0, \\
E \alpha+D \beta+4 \gamma^{2}(B \beta-C \alpha)+\gamma^{2}\left(7-3 \alpha^{2}\right) & =0 .
\end{aligned}
$$

Here, the following notation is used: 


$$
\begin{aligned}
& B=\varkappa_{1}^{-2} \dot{\varkappa}_{1}, \quad C=\varkappa_{1}^{-1} \varkappa_{2}, \\
& D=\varkappa_{1}^{-3}\left(2 \dot{\varkappa}_{1} \varkappa_{2}+\varkappa_{1} \dot{\varkappa}_{2}\right), \\
& E=\varkappa_{1}^{-3}\left(\ddot{\varkappa}_{1}+\varkappa_{1}^{3}-\varkappa_{1} \varkappa_{2}^{2}\right) .
\end{aligned}
$$

Conditions (23), (24) determine unknowns $\alpha, \beta, \gamma$ of decomposition (21) in terms of derivatives of trajectory. The system (23) is overcomplete because three unknowns are subjected to four equations.

The quantities $\alpha, \beta$ are easily expressed from the first and second equations of the system (23),

$$
\gamma=\sqrt{-\alpha}, \quad \beta=\frac{B \alpha}{3 \alpha-C} .
$$

Substituting this solution into the third and fourth relations (23), we get two polynomial constraints for a remaining unknown $\alpha$,

$$
\begin{gathered}
P_{1}(\alpha)=9 \alpha^{3}+9 C \alpha^{2}-\left(4 B^{2}+4 C^{2}-3 E+21\right) \alpha \\
+B D-E C+7 C=0 . \\
P_{2}(\alpha)=9 \alpha^{4}-6 C \alpha^{3}+\left(B^{2}+C^{2}-9\right) \alpha^{2} \\
+6 C \alpha-C^{2}=0 .
\end{gathered}
$$

Relation (27) determines the unknown $\alpha$ in terms of derivatives of trajectory up to third order. The explicit representation for $\alpha$ can be found by application of standard solution to cubic equation (26), for example Cardano formula. The only negative root is relevant because of condition (25). We do not provide this solution because the system (26), (27) admits a simpler representation without radicals. We give it in the next paragraph. Relation (26) is another restriction for unknown $\alpha$. Since both the conditions (26), (27) are consequences of cylinder equation (6), they has to be satisfied simultaneously. So, $\alpha$ is a common root of polynomials $P_{1}(\alpha)$ and $P_{2}(\alpha)$. Two different polynomials have a common root if and only if their resultant with respect to the variable $\alpha$ vanishes,

$$
\operatorname{Res}_{\alpha}\left(P_{1}(\alpha), P_{2}(\alpha)\right)=0
$$

This is consistency condition for the system (26), (27). By construction, the resultant is a polynomial in the coefficients of polynomials (26), (27), being functions of curvature and torsion of world line, and their derivatives. This resultant is a differential equation, being satisfied by the cylindrical curves. It involves derivatives of the path up to fourth order.

Let us now find explicit solution for $\alpha$ and representation for resultant (28). Introduce special notation for combinations of derivatives of curvature and torsion,

$$
\begin{aligned}
& F=-\left(4 B^{2}+4 C^{2}-3 E+21\right) / 9, \\
& G=(B D-E C+7 C) / 9, \\
& H=B^{2}+C^{2}-9 .
\end{aligned}
$$

Here, $F, G, H$ can be considered as alternative combinations absorbing invariants of trajectory and their derivatives. In terms of quantities $F, G, H$ Eqs. (26), (27) take the most simple form:

$$
\begin{gathered}
P_{1}(\alpha)=\alpha^{3}+C \alpha^{2}+F \alpha+G=0, \\
P_{2}(\alpha)=9 \alpha^{4}-6 C \alpha^{3}+H \alpha^{2}+6 C \alpha-C^{2}=0 .
\end{gathered}
$$

The consistency condition in terms of resultant of polynomials (30), (30) reads

$$
\begin{aligned}
\operatorname{res}_{\alpha}\left(P_{1}(\alpha), P_{2}(\alpha)\right)= & 81 F^{2} G^{2} H-18 F G^{2} H^{2}-15 C^{4} F^{2} H+18 C^{2} F^{3} H-C^{2} F^{2} H^{2}+486 C F G^{3} \\
& -324 C G^{3} H+270 C^{5} F G-162 C^{3} F^{2} G+1458 C^{2} F G^{2}+18 C^{3} G H+189 C^{2} G^{2} H+4 C^{4} F H \\
& -1134 C^{3} F G+15 C^{2} G^{2} H^{2}+2 C^{3} G H^{2}+30 C^{5} G H-486 C F^{3} G+G^{2} H^{3}-120 C^{3} F G H+90 C^{2} F G^{2} H \\
& -6 C F G H^{2}+108 C F^{2} G H-216 C^{3} G+972 C^{2} G^{2}-36 C^{4} F+504 C^{5} G+540 C^{4} G^{2}-216 C^{4} F^{2} \\
& +36 C^{6} F+540 C^{3} G^{3}-81 C^{2} F^{4}-90 C^{4} F^{3}-1458 C G^{3}+C^{6} H-7 C^{6}+729 G^{4}+15 C^{8}=0 .
\end{aligned}
$$

The common root of (30), (31) can be determined by the Euclid algorithm. Assuming that this root is simple (i.e., the curve lies on a single cylinder), we obtain

$$
\alpha=-\frac{U}{V},
$$

where

$$
\begin{aligned}
U= & 225 F C^{2}-6 C G H+15 C^{2} F H-216 G F G \\
& -90 C^{3} G+90 C^{2} F^{2}-75 C^{4}-5 C^{2} H+81 G^{2} \\
& -108 C G+36 C^{2}+81 F^{3}+F H^{2}-18 F^{2} H, \\
V= & C^{3} H+G H^{2}-18 F G H+81 F^{2} G \\
& -135 C G^{2}-6 C^{3}+15 C^{2} G H+15 C^{5} \\
& -24 C^{3} F+90 F C^{2} G+99 C^{2} G .
\end{aligned}
$$


Relation (32) determines the equation of motion for cylindrical curves. Formulas (33), (34), (35) determine a solution to Eqs. (26), (27) with respect to unknown $\alpha$.

In terms of auxiliary quantity $\alpha$ (33) the solution for the momentum $p$ and vector $n$ reads

$$
\begin{gathered}
p=\sqrt{-\operatorname{sign}(\sigma) \sigma \varkappa_{1} \alpha}\left(\dot{x}-\frac{B \alpha}{\varkappa_{1}(3 \alpha-C)} \ddot{x}+\frac{\alpha}{\varkappa_{1}}[\dot{x}, \ddot{x}]\right), \\
n=\frac{\sigma}{\varkappa_{1}}\left(\alpha \ddot{x}+\frac{B \alpha}{3 \alpha-C}[\dot{x}, \ddot{x}]\right) .
\end{gathered}
$$

Relations (13), (6) determine the cylinder parameters $v, a$,

$$
\begin{aligned}
v= & \sigma \alpha\left[\left((x, \dot{x}) \varkappa_{1}+(x, \dot{x}, \ddot{x}) \varkappa_{1} \alpha-(x, \ddot{x}) B \alpha\right) \dot{x}\right. \\
+ & \frac{1}{\varkappa_{1}}\left(1-\frac{B \alpha}{C-3 \alpha}\left((x, \dot{x}) \varkappa_{1}+(x, \dot{x}, \ddot{x}) \alpha\right)\right. \\
- & \left.\frac{(x, \ddot{x}) B^{2} \alpha^{2}}{C-3 \alpha}\right) \ddot{x}-\frac{1}{\varkappa_{1}}\left((x, \dot{x}) \varkappa_{1} \alpha+(x, \dot{x}, \ddot{x}) \alpha^{2}\right. \\
+ & \left.\left.\frac{B}{C-3 \alpha}\left(1-(x, \ddot{x}) \alpha^{2}\right)\right)[\dot{x}, \ddot{x}]\right], \\
a= & \frac{\sigma \alpha}{\varkappa_{1}}\left[\left((x, \dot{x}) \varkappa_{1}+(x, \dot{x}, \ddot{x}) \alpha+\frac{(x, \ddot{x}) B \alpha}{C-3 \alpha}\right)^{2}\right. \\
& \left.+\frac{(x, \dot{x}, \ddot{x}) B}{C-3 \alpha}-2(x, \ddot{x})\right] .
\end{aligned}
$$

The solution for the total angular momentum $J$ (9) reads

$$
\begin{aligned}
J= & {\left[\frac{(x, p)^{2} \varkappa_{1} \alpha-\sigma}{2 \sqrt{-\operatorname{sign}(\sigma) \sigma \varkappa_{1} \alpha}}+\frac{a+\varrho}{2} \sqrt{-\frac{\alpha \varkappa_{1}}{\sigma}}\right] \dot{x} } \\
& +\frac{\alpha}{\varkappa_{1}}\left[\left((x, p)+\frac{B}{C-3 \alpha} \frac{(x, p)^{2} \varkappa_{1} \alpha+\sigma}{2 \sqrt{-\operatorname{sign}(\sigma) \sigma \varkappa_{1} \alpha}}\right)\right. \\
& \left.+\frac{B}{(C-3 \alpha)} \frac{a+\varrho}{2} \sqrt{-\frac{\alpha \varkappa_{1}}{\sigma}}\right] \ddot{x} \\
& +\frac{\alpha}{\varkappa_{1}}\left[\left(\frac{(x, p)^{2} \varkappa_{1} \alpha+\sigma}{2 \sqrt{-\operatorname{sign}(\sigma) \sigma \varkappa_{1} \alpha}}-\frac{B(x, p)}{C-3 \alpha}\right)+\sqrt{-\frac{\alpha \varkappa_{1}}{\sigma}}\right][\dot{x}, \ddot{x}] .
\end{aligned}
$$

The solution uses auxiliary vector $e(10)$ with $p$ and $v$ being given by (36), (38), respectively. Relations (36), (40) determine the particle momentum $p$ and particle total angular momentum $J$ (hence, the cylinder parameters $v, a)$ in terms of derivatives of classical trajectory. The description of the particle state is purely geometrical because no internal variables are involved in (36), (40).

Equation (32) has two obvious gauge symmetries: reparametrization and translations along the cylinder axis,

$$
\delta_{\xi} x=\dot{x} \xi, \quad \delta_{\eta} x=p \eta .
$$

In the last case, the vector $p$ is considered as the function of derivatives of trajectory (36). The gauge transformations are independent for general timelike lines because the vectors $\dot{x}, p$ are nonzero and noncollinear. The vector $p$ is nonzero for spinning particle with nonzero helicity [see conditions (1)]. The velocity vector $\dot{x} \neq 0$ is nonzero because the spinning particle is timelike and casual. The vectors $\dot{x}$ and $p$ are not collinear because $p$ is lightlike and $\dot{x}$ is timelike. The tangent space to the cylinder (6) is twodimensional, so the gauge symmetry is sufficient to connect each pair of timelike curves that lie on one and the same worldsheet. This result confirms the relationship between the general timelike cylindrical curves and spinning particle trajectories. For $p=(1,0,1), J=(0,0,1), \varrho=1$, the particular example of timelike paths is given by the curves

$$
\begin{aligned}
& x^{0}=t^{3}+(\theta+1) t, \quad x^{1}=-0.5 t^{2}, \\
& x^{2}=t^{3}+\theta t,
\end{aligned}
$$

where $\theta>-0.5$ and $t$ is an arbitrary (not natural) parameter on the curve. The trajectory (42) for $\theta=1$ is shown in the Fig. 2.

Now, we can summarize the results of the subsection. We have associated the timelike spinning particle trajectories as curves on parabolic cylinders. We have shown that these curves are solutions to the fourth-order differential equation (32). Equations (36), (40) determine the particle momentum and total angular momentum in terms of derivatives of trajectory. The representation for $p, J$ does not involve internal variables, so the particle state description is purely geometrical. Equations (41) determine the gauge transformations for spinning particle trajectories. In Sec. IV we show that the geometrical equation of motion

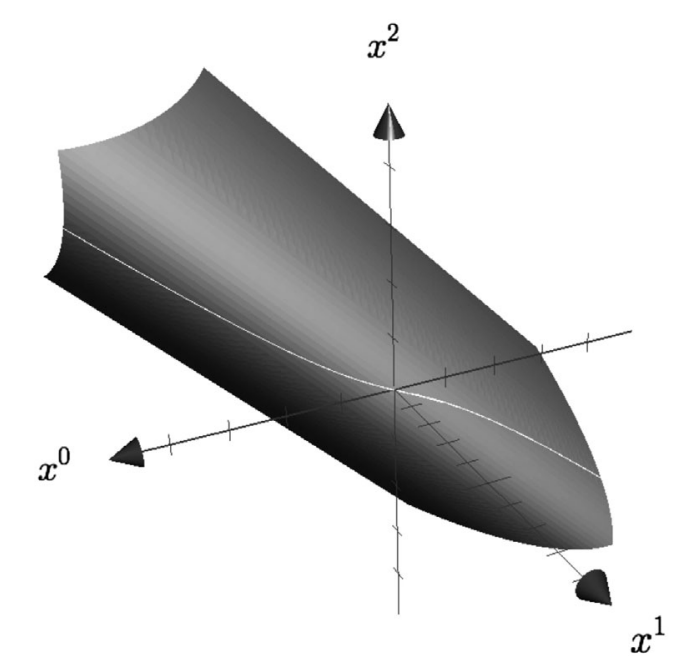

FIG. 2. Timelike curve (42) for $\theta=1$ on parabolic cylinder with lightlike axis. The step along the axes equals 1 . 
follow from the previously known model. This ensures that this model describes spinning particle with continuous helicity at the quantum level.

\section{Isotropic paths on the worldsheet}

Let $x(\tau)$ be an lightlike curve, so the length of the tangent vector is equal to zero,

$$
(\dot{x}, \dot{x})=0 .
$$

The natural parameter on the curve (so-called pseudo-arc length) is determined by the condition

$$
(\ddot{x}, \ddot{x})=1 .
$$

The natural parameter $\tau$ is well defined on an arbitrary isotropic curve which is not a straight line. This does not restrict generality because all the rectilinear paths on the parabolic cylinder with lightlike axis are cylinder elements, being atypical curves. The atypical curves are excluded from our consideration.

The Frenet-Serret moving frame, being associated with the isotropic curve $x(\tau)$, reads

$$
e_{0}=\dot{x}, \quad e_{1}=\ddot{x}, \quad e_{2}=-\dddot{x}-\frac{1}{2}(\dddot{x}, \dddot{x}) \dot{x} .
$$

The basis $e_{a}, a=0,1,2$ includes two lightlike vectors $e_{0}$ and $e_{2}$ with normalized scalar product, and normalized timelike vector $e_{1}$,

$$
\begin{aligned}
& \left(e_{0}, e_{2}\right)=\left(e_{1}, e_{1}\right)=1, \\
& \left(e_{0}, e_{0}\right)=\left(e_{2}, e_{2}\right)=\left(e_{0}, e_{1}\right)=\left(e_{1}, e_{2}\right)=0 .
\end{aligned}
$$

The basis (45) is well-defined for each lightlike curve, which is not a straight line.

The Frenet-Serret formulas for the lightlike curve $x(\tau)$ read

$$
\dot{e}_{0}=e_{1}, \quad \dot{e}_{1}=\varkappa e_{0}-e_{2}, \quad \dot{e}_{2}=-\varkappa e_{1} .
$$

The lightlike curve is characterized by a single invariant $\varkappa$, which includes third derivatives of the path

$$
\varkappa=-\frac{1}{2}(\dddot{x}, \dddot{x}) .
$$

The quantity $\varkappa$ can be interpreted as some special analog of curvature, even though its geometrical meaning is slightly different. As we will see below, the condition $x=0$ selects the lightlike curves on the parabolic cylinder. The representation of derivatives of trajectory in terms of curvature $x$ reads

$$
\begin{aligned}
\dot{x} & =e_{0}, \quad \dddot{x}=e_{1}, \\
\dddot{x} & =\varkappa e_{0}-e_{2}, \quad \dddot{x}=\dot{x} e_{0}+2 \varkappa e_{1} .
\end{aligned}
$$

As usual, this representation involves invariants of trajectory up to fourth order $\varkappa, \dot{\varkappa}$.

We seek for unknown vectors $p, n$, being determined by the conditions (12), (14) in the following form:

$$
\begin{aligned}
& p=\gamma \sqrt{\operatorname{sign}(\sigma) \sigma}\left(\frac{1}{2} \beta^{2} e_{0}+\beta e_{1}-e_{2}\right), \\
& n= \pm \sigma\left(\beta e_{0}+e_{1}\right)+\alpha p
\end{aligned}
$$

where $\alpha, \beta, \gamma$ are new unknowns. The quantity $\alpha$ has the dimension of angular momentum. The quantity $\beta$ has the dimension of inverse square root of length. The quantity $\gamma$ is dimensionless. Moreover, we assume $\gamma>0$ in order to meet the condition $p^{0}>0$. The sign of \pm determines relative orientation of vector $p$ with respect to the FrenetSerret frame (45).

On substituting representation (50) into (12), we arrive at the following system of algebraic equations for $\alpha, \beta$, and $\gamma$ :

$$
\begin{aligned}
\alpha \gamma & =0, \\
\pm 1+\gamma^{2}+\frac{\alpha \beta \gamma}{\sqrt{\sigma}} & =0, \\
\beta\left( \pm 1-3 \gamma^{2}-\frac{\alpha \beta \gamma}{2 \sqrt{\sigma}}\right)+\varkappa \frac{\alpha \gamma}{\sqrt{\sigma}} & =0, \\
2 \varkappa\left( \pm 1+2 \gamma^{2}+\frac{\alpha \beta \gamma}{\sqrt{\sigma}}\right)+5 \gamma^{2} \beta^{2}-\dot{\varkappa} \frac{\alpha \gamma}{\sqrt{\sigma}} & =0 .
\end{aligned}
$$

The solution to these equations eventually reads

$$
\alpha=\beta=0, \quad \gamma=1 .
$$

For the vectors $p$ and $n$, we find

$$
p=\sqrt{\operatorname{sign}(\sigma) \sigma} \dddot{x}, \quad n=-\sigma \ddot{x} .
$$

The cylinder parameters $v, a$ read

$$
\begin{aligned}
& v=-\sigma(\ddot{x}+(x, \dddot{x}) \dddot{x}), \\
& a=\sigma\left(2(x, \ddot{x})+(x, \dddot{x})^{2}\right) .
\end{aligned}
$$

The representation for the total angular momentum $J$ reads

$$
\begin{aligned}
J= & -\sqrt{\operatorname{sign}(\sigma) \sigma}[\dot{x}+(x, \dddot{x}) \ddot{x} \\
& \left.-\left((x, \ddot{x})+\frac{\varrho}{2 \sigma}\right) \dddot{x}\right] .
\end{aligned}
$$

The solution uses explicit representation for the auxiliary vector $e(10)$ 


$$
e=-\frac{1}{\sqrt{\operatorname{sign}(\sigma) \sigma}}\left(\dot{x}+(x, \dddot{x}) \ddot{x}+\frac{(x, \dddot{x})^{2}}{2} \dddot{x}\right) .
$$

The system (51) has a consistency condition,

$$
\varkappa=0 \text {. }
$$

From the differential geometry of the curves, this equation means that the lightlike path on the parabolic cylinder have zero curvature. This fact means that the lightlike paths on the parabolic cylinder with lightlike axis (6) are indeed the curves with zero curvature. The complete set of equations for the cylindrical paths includes zero curvature condition (57) and the lightlike condition for the particle velocity (43),

$$
(\dddot{x}, \dddot{x})=0, \quad(\dot{x}, \dot{x})=0 .
$$

The first equations in this system has the third order in derivatives, and the second one has the first order. Equation (58) has a single gauge symmetry, being a reparametrization,

$$
\delta_{\xi} x=\dot{x} \xi
$$

where $\xi=\xi(\tau)$ is an arbitrary function of proper time.

The identification between the lightlike curves on parabolic cylinders and the trajectories of spinning particles suggests that the all the parameters of trajectory are determined by the momentum and total angular momentum. As we will see, this is not true. The general solution to Eq. (58) reads

$$
x(\tau)=\frac{1}{6} a \tau^{3}+\frac{1}{2} b \tau^{2}+c \tau+d .
$$

The quantity $\tau$ is a natural parameter on the lightlike curve. The Cauchy data are constant vectors $a, b, c, d$ that are subject to conditions

$$
\begin{aligned}
-(a, c) & =(b, b)=1, \\
(a, a) & =(c, c)=(a, b)=(b, c)=0 .
\end{aligned}
$$

We also assume that $(d, a)=0$. If this is not true, then we make a reparametrization $\tau \mapsto \tau+\gamma$ with appropriate $\gamma$. The curve (60) lies on the worldsheet (6) if

$$
\begin{array}{ll}
a=\frac{1}{\sqrt{\operatorname{sign}(\sigma) \sigma}} p, \quad b=\frac{1}{\sigma}[p, J], \\
c=-\sqrt{\operatorname{sign}(\sigma) \sigma} e, \quad(d, b)=\frac{1}{2 \sigma}((J, J)-\varrho) .
\end{array}
$$

The particle state determines the parameters of trajectory if these equations can be solved with respect to $a, b, c, d$. This is not possible because the solution for $d$ has ambiguity,

$$
d=\frac{1}{2 \sigma^{2}}((J, J)-\varrho)[p, J]+\frac{\lambda}{p^{0}} p
$$

Here, $\lambda \in \mathbb{R}$, and $p^{0}$ is the time component of momentum $p$. The quantity $\lambda$ controls parallel shifts of path along the symmetry axis of cylinder. It is an additional data, being independent of $p$ and $J$. Thus the position of general lightlike cylindrical curve is determined by five parameters. The theory (58) cannot be a dynamical system on the continuous helicity co-orbit (1), which has two physical degrees of freedom (four physical polarizations).

Figure 3 shows two particular lightlike curves lying on the parabolic cylinder with $p=(1,0,1), J=(0,0,1)$, $Q=1$. The curve passing through the origin corresponds to $\lambda=0$. The second curve corresponds to $\lambda=2$. These curves are not connected by a gauge transformation.

The results of the subsection demonstrate that the lightlike world lines are not admissible classical trajectories of relativistic spinning particle with continuous helicity. In particular, no geometrical model of continuous helicity spinning particle can be constructed with lightlike trajectories. The problem of lightlike curves has no analog in massive case, where the geometrical models of relativistic particles with lightlike lines are known for a long time [30-32]. Our no-go result for continuous helicity particle suggests that the presence of lightlike trajectories is a feature of massive models.

\section{HAMILTON'S FORMALISM}

In the articles [6], the equations of motion of irreducible spinning particles has been derived from the action functional involving extra variables, being internal space coordinates. In the context of current research the model [6] is relevant. The paper considers the massive particle, but the action functional admits a smooth continuous helicity

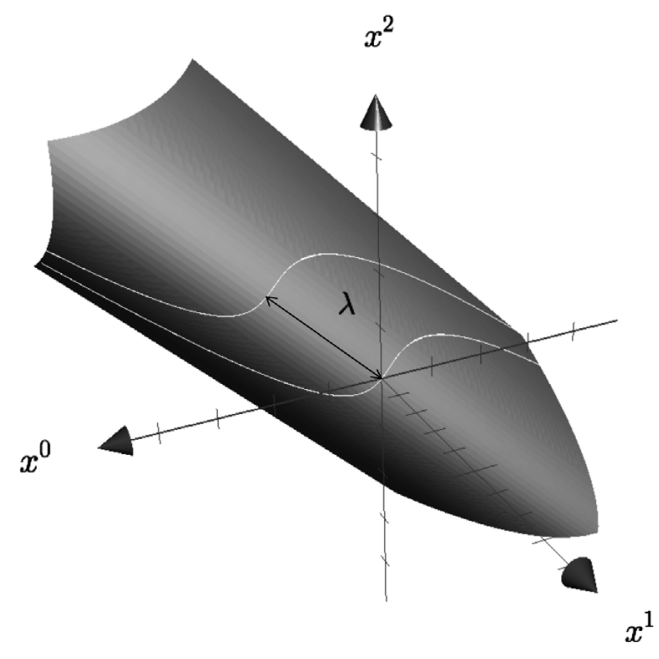

FIG. 3. Lightlike curves on parabolic cylinder with lightlike axis. The step along the axes equals 1 . 
limit $m \rightarrow 0, m s \rightarrow \sigma$. In this section, we demonstrate that the equations of motion for cylindrical curves follow form the least action principle of the work [6]. For reasons of simplicity, we consider the case of spacelike or lightlike spin vector. The accessory parameter $\varrho$ is determined by the rule

$$
(M, M)=\alpha^{2},
$$

which corresponds to the identification $\varrho=\alpha^{2}$. The case of lightlike spin vector can be considered in the similar way. We leave the details to the reader.

The equation of the worldsheet can be equivalently rewritten in the following vector form:

$$
x=(x, e) p+(x, p) e-\frac{(x, p)^{2}+a}{2 \sigma^{2}} v .
$$

The vector equation has the same valuable information about the particle position as a scalar relation because it has only one independent component. The scalar multiplication of left- and right-hand sides of Eq. (65) on $p, e$ leads to identity. The only nontrivial consequence of relation appears after multiplication of both sides of the equation by $v$, and it gives the (6). Relation (65) can be considered as the solution to the worldsheet equation in the parametric form. In this setting, the functions $(x, p),(x, e)$ serve as local coordinates on the cylinder. Once the spinning particle travels the path on the worldsheet, the quantities $(x, p),(x, e)$ are arbitrary functions of proper time, being restricted by the causality condition. In what follows, we assume that the causal trajectories are considered.

Relation (65) determines the dynamics of spinning particle. Differentiating by the proper time, we obtain

$$
\dot{x}=(\dot{x}, e) p+(\dot{x}, p)\left(e-\frac{(x, p)}{\sigma^{2}} v\right),
$$

where $(\dot{x}, p),(\dot{x}, e)$ are arbitrary functions. The quantities $(\dot{x}, p),(\dot{x}, e)$ have sense of velocities of generalized coordinates $(x, e),(x, p)$ on the worldsheet. Equation (66) should be complimented by the conservation law for momentum and total angular momentum,

$$
\dot{p}=\dot{v}=\dot{e}=0,
$$

and the constraints for the vectors $p, v, e$,

$$
\begin{aligned}
(p, p) & =(e, e)=(e, v)=0, \\
(e, p)-1 & =0, \quad(v, v)-\sigma^{2}=0 .
\end{aligned}
$$

The system (66), (67), (68) determine the class of cylindrical curves by obvious reasons. Equations (67), (68) tell us that the vectors $p, v, e$ are integrals of motion subjected to constraints (68). After that the integration of differential
Eq. (66) gives (65). The quantity $a$ appears as the constant of integration. In so doing, the spinning particle travels along the cylindrical path if and only if equations are satisfied (66), (67), (68).

The system (66), (67), (68) does not follow from the least action principle of dynamical variables $x, p, v, e,(\dot{x}, p)$, $(\dot{x}, e)$ because 14 quantities are subjected to 18 evolutionary equations and constraints. To construct the variational principle, we solve constraints (68) using the lightlike vector $\xi$ with normalized 0 component,

$$
\xi=(1, \sin \varphi, \cos \varphi) .
$$

The new dynamical variable $\varphi$ can be considered as angular variable in the internal space, being a circle. This corresponds to the configuration space of spinning particle $\mathbb{R}^{1,2} \times \mathbb{S}^{1}$. By definition, we put

$$
\begin{aligned}
& v=\sigma \frac{[\xi, p]}{(\xi, p)}-(\alpha+(x, p)) p, \\
& e=\frac{\xi}{(\xi, p)}+\frac{(x, p)+\alpha}{\sigma(\xi, p)}[\xi, p]-\frac{((x, p)+\alpha)^{2}}{2 \sigma^{2}} p,
\end{aligned}
$$

where $p$ is the momentum, being lightlike vector. Relation (70), (70) automatically meets all the constraints (68) involving $v$ and $e$. The only reaming constraint is the mass shell condition for the particle momentum

$$
(p, p)=0 .
$$

In terms of dynamical variables $x, p, \xi$ Eqs. (66), (67), (68) take the following form:

$$
\begin{gathered}
\dot{x}=\left((\dot{x}, e)+\frac{(x, p)^{2}-\alpha^{2}}{2 \sigma^{2}}(\dot{x}, p)\right) p \\
+(\dot{x}, p)\left(\frac{\xi}{(\xi, p)}+\frac{\alpha}{\sigma} \frac{[\xi, p]}{(\xi, p)}\right), \\
\frac{d}{d \tau}\left(\sigma \frac{[\xi, p]}{(\xi, p)}-(\alpha+(x, p)) p\right)=0, \\
\dot{p}=0, \quad(p, p)=0 .
\end{gathered}
$$

We dot not write out the consequences of the condition $\dot{e}=0$ because the quantity is completely determined by $p$ and $v$. Relations (72), (73), (74) have clear physical sense. Equation (72) determines the evolution of the particle position. Equation (74) tells us that the vector $p$ conserves, and it is lightlike. Equation (73) expresses a single independent relation because the vector $v$ has a single independent component. It has a consequence

$$
(\dot{x}, p)=\frac{\sigma \dot{\varphi}}{(\xi, p)} .
$$


On substituting this expression for $(\dot{x}, p)$ into (72), we obtain the system of two vector equations (72), (74) for the dynamical variables $x, p, \varphi$.

Relations (72), (74), (75) follow from the least action principle for the functional

$$
\begin{aligned}
S= & \int\left\{(p, \dot{x})+\frac{\sigma}{(\xi, p)} \dot{\varphi}\right. \\
& \left.+\alpha \frac{\left(\partial_{\varphi} \xi, p\right)}{(\xi, p)} \dot{\varphi}-\frac{\lambda}{2}(p, p)\right\} d \tau .
\end{aligned}
$$

The dynamical variables are the particle position $x$, momentum $p$, angular variable $\varphi$, and Lagrange multiplier $\lambda$. Relations (72), (74), (75) appear as the variational derivatives with respect to the dynamical variables $x, p$, $\lambda$. Taking the Lagrange derivative with respect to $p$, we obtain Eqs. (72), (75),

$$
\begin{aligned}
\dot{x}= & \left((\dot{x}, e)+\frac{(x, p)^{2}-\alpha^{2}}{2 \sigma^{2}} \frac{\sigma \dot{\varphi}}{(\xi, p)}\right) p \\
& +\frac{\sigma \dot{\varphi}}{(\xi, p)}\left(\frac{\xi}{(\xi, p)}+\frac{\alpha}{\sigma} \frac{[\xi, p]}{(\xi, p)}\right) .
\end{aligned}
$$

Taking the Lagrange derivative with respect to $x$ and $\lambda$, we get (74). The variation of action (76) with respect to $\varphi$ does not lead to a new independent dynamical equation because of gauge identity

$$
(\xi, p) \frac{\delta S}{\delta \varphi}+\frac{\sigma \dot{\varphi}}{(\xi, p)} \frac{\delta S}{\delta x}=0
$$

This proves the variational principle for the cylindrical curves.

It remains to verify that the quantization of the classical model (76) corresponds to the irreducible representation with helicity $\sigma$ and accessory parameter $\alpha^{2}$. The fact is nontrivial because the particles of continuous helicity follow one and the same paths irrespectively to the value of the representation parameters. The total angular momentum vector reads

$$
J=[x, p]+\left(\frac{\sigma}{(\xi, p)}+\frac{\alpha\left(\partial_{\varphi} \xi, p\right)}{(\xi, p)}\right) \xi-\alpha \partial_{\varphi} \xi .
$$

One can see that the vector $J$ meets spin shell condition

$$
(p, J) \equiv \sigma
$$

The spin angular momentum reads

$$
M=\left(\frac{\sigma}{(\xi, p)}+\frac{\alpha\left(\partial_{\varphi} \xi, p\right)}{(\xi, p)}\right) \xi-\alpha \partial_{\varphi} \xi
$$

It is easy to see that condition (64) is true. This result ensures that the geometrical equations of motion for cylindrical lines admit equivalent variational formulation with the auxiliary variables. In its own turn, the variational model can be quantized in a way that corresponds to the continuous helicity representation of the Poincare group.

\section{MASSLESS PARTICLE}

The massless co-orbit is determined by the relations (1) with $\sigma=0$. The momentum $p$ and total angular momentum $J$ of massless particle are subjected to following mass-shell and spin-shell conditions,

$$
(p, p)=0, \quad(p, J)=0 .
$$

These relations are inconsistent for timelike $J$, so we assume that the norm of $J$ is nonnegative throughout the section. The spin vector $M$ is determined by the rule (2). Similarly to $J$, the vector $M$ is lightlike or spacelike. The accessory parameter $\varrho=\alpha^{2}$ is determined by the relation

$$
(M, M)=\alpha^{2} .
$$

Now, we can discuss the structure of spinning particle worldsheet. Equation (82) has a consequence,

$$
[J, p]=(J, J)^{\frac{1}{2}} p .
$$

It means that the quantity $v(7)$ and momentum $p$ are collinear, while the norm of $J$ determines the aspect ratio. With account of (84), the equation of the worldsheet of spinning particle eventually reads

$$
\begin{aligned}
(J & -[x, p])^{2}-\alpha^{2} \\
& =\left((x, p)+(J, J)^{\frac{1}{2}}+\alpha\right)\left((x, p)+(J, J)^{\frac{1}{2}}-\alpha\right)=0 .
\end{aligned}
$$

The formula determines the pair of parallel hyperplanes with the normal $p$. The quantity $\alpha$ controls the distance between hyperplanes. The worldsheet is path connected if $\alpha=0$. In the latter case, we have a single hyperplane,

$$
(x, p)+(J, J)^{\frac{1}{2}}=0 .
$$

Here, the vector $p$ serves as the normal, while the norm of $J$ determines the distance between the hyperplane (86) and origin.

Equations (85) and (86) tell us that the positions of massless spinning particle are localized on the hyperplanes, whose position in Minkowski space is defined by the values of momentum and total angular momentum. This fact has been observed previously in chiral fermion model $[33,34]$ earlier. Our result shows that the planar motion has no alternative for the massless particle irrespectively to any 
specifics of the model. In particular, the torsion of the spinning particle path must be zero in all the instances,

$$
(\dot{x}, \ddot{x}, \dddot{x})=0 .
$$

Unfortunately, this equation contains only partial information about the model dynamics. The number of independent parameters labeling the particular hypersurface in the set (85), (86) is less than the co-orbit dimension. In the case of two hyperplanes (85), the position of the worldsheet is determined by three parameters: the lightlike vector $p$, and the norm of total angular momentum $(J, J)^{\frac{1}{2}}$. In the case of a single hyperplane (86), only the ratio $p /(J, J)^{\frac{1}{2}}$ is relevant. It involves only two initial data in independent way. The dimension of co-orbit (82) equals four in all the instances. The extra dynamical degrees of freedom have no geometrical description in terms of worldsheet formalism, and they require introduction of internal variables. This result means that worldsheet concept cannot be used for construction of geometric model of massless spinning particle. On the other hand, the spinning particles must be planar curves in every irreducible spinning particle theory. As it has been mentioned above, this condition is satisfied for previously known models.

\section{CONCLUSION}

In the current article, the recently proposed idea of characterizing the classical spinning particle dynamics by the worldsheet, rather than the world line [26], has been applied to the problem of description of dynamics of irreducible spinning particle with continuous helicity. It has been shown that the admissible classical positions of the particle lie on parabolic hypercylinder in Minkowski space irrespectively to any specifics of the model. The position of the hypercylinder is determined by the values of momentum and total angular momentum. The focal distance is determined by helicity. The classical trajectories of the spinning particle are given by causal cylindrical lines. Assuming that all the trajectories belonging to the same cylinder are connected by gauge transformation, we have derived an ordinary differential equation describing the general cylindrical lines with timelike tangent vector. These equations of motion are purely geometrical, and they involve invariants of the classical path including the derivatives up to the fourth order. The momentum and total angular momentum are expressed as the functions of trajectory. To our best knowledge, geometrical equations of motion, not involving any extra variables, have been previously unknown for the continuous helicity particle.

We have paid the particular attention to the class of lightlike cylindrical lines. It has been shown that the cylindrical lightlike trajectory either a straight line (representing the cylinder element) or the curve with zero (lightlike) curvature. Unlike the massive case, no lightlike curves can serve as physically acceptable trajectories of spinning particles. The lines lie on infinite number of parabolic cylinders with one and the same direction of axis. These trajectories do not determine the state of the particle in unambiguous way. As for zero curvature paths, their position in space-time involves besides the momentum and total angular momentum an extra initial data. Having an extra degree of freedom, the theory of lightlike cylindrical curves cannot be considered as the a dynamical system on the continuous helicity co-orbit. As the spinning particles are dynamical systems on the co-orbit, this theory cannot describe the motion of spinning particle.

We have proven that the geometric equations of motion for cylindrical curves can be derived from the least action principle. This is important from several viewpoints. First, the concept of irreducible spinning particle suggests that the model can be quantized, while its quantization corresponds to the irreducible representation of the Poincare group. The variational principle provides the way to constructing the quantum theory. Second, the variational principle shows that our results are consistent with the previous studies. We explicitly demonstrate that the differential equations for cylindrical curves follow from the action functional of the work [6], and vice versa. The quantization of this model do correspond to the continuous helicity representation. In all the cases, the variational principle involves extra variables having sense of coordinates in internal space.

The studies of the spinning particle worldsheet concept can be continued in several directions. One of the interesting issues is the geometry trajectories of spinning particle in the external electromagnetic and gravitational field. The article [29] tells us that the worldsheet of massive particle in electromagnetic field is a cylindrical hypersurface, whose radius is fixed by the representation. The worldsheets of continuous helicity may have much more interesting geometry because the sections of parabolic cylinder are not compact. The couplings between the particle traveling a cylindrical path and external field are expected to be nonlocal, while the nonlocality is controlled by the helicity. Expanding these equations in helicity, we will obtain approximate equations describing cylindrical trajectories of continuous helicity spinning particles. The leading orders of these equations will serve as the analogs of Frenkel [1] and Mathisson-Papapetrou [40,41] models.

\section{ACKNOWLEDGEMENTS}

The authors thank A. A. Sharapov for valuable discussions of this work. We also thank V. A. Abakumova and E.V. Koryukina for plotting figures. The work was supported by the Tomsk State University Development Programme (Priority-2030). The work of S.L.L. and I. A. R. was supported by Foundation for the Advancement of Theoretical Physics and Mathematics "BASIS." 
[1] J. Frenkel, Die Elektrodynamik des rotierenden Elektrons, Z. Phys. 37, 243 (1926).

[2] H. C. Corben, Classical and Quantum Theories of Spinning Particles (Holden-Day, Inc, San Francisco, 1968).

[3] A. Frydryszak, in From Field Theory To Quantum Group (World Scientific Publishing, Singapore, 1996), pp. 151-172.

[4] S. L. Lyakhovich, A. Yu. Segal, and A. A. Sharapov, Universal model of a $D=4$ spinning particle, Phys. Rev. D 54, 5223 (1996).

[5] A. Silenko, Zitterbewegung of Bosons, Phys. Part. Nucl. Lett. 17, 116 (2020).

[6] I. V. Gorbunov, S. M. Kuzenko, and S. L. Lyakhovich, On the minimal model of anyons, Int. J. Mod. Phys. A 12, 4199 (1997).

[7] K. B. Alkalaev and S. L. Lyakhovich, On the consistency problem of interactions of $(2+1)$ massive spinning particle, Mod. Phys. Lett. A 14, 2727 (1999).

[8] S. L. Lyakhovich, A. A. Sharapov, and K. M. Shekhter, Spinning particle in six dimensions, J. Math. Phys. (N.Y.) 38, 4086 (1997).

[9] S. L. Lyakhovich, A. A. Sharapov, and K. M. Shekhter, Massive spinning particle in any dimension. (I) Integer spins, Nucl. Phys. B537, 640 (1999).

[10] S. L. Lyakhovich, A. A. Sharapov, and K. M. Shekhter, A uniform model of the massive spinning particle in any dimension, Int. J. Mod. Phys. A 15, 4287 (2000).

[11] S. M. Kuzenko, S. L. Lyakhovich, A. Yu. Segal, and A. A. Sharapov, Massive spinning particle on anti-de Sitter space, Int. J. Mod. Phys. A 11, 3307 (1996).

[12] D. V. Karlovets and I. P. Ivanov, Detecting Transition Radiation from a Magnetic Moment, Phys. Rev. Lett. 110, 264801 (2013).

[13] J. H. Field, E. Picasso, and F. Combley, Tests of fundamental physical theories from measurements on free charged leptons, Sov. Phys. Usp. 22, 199 (1979).

[14] G. H. Hoffstaetter, H. S. Dumasand, and J. A. Ellison, Adiabatic invariance of spin-orbit motion in accelerator, Phys. Rev. ST Accel. Beams 9, 014001 (2006).

[15] M. I. Dyakonov, Magnetoresistance due to Edge Spin Accumulation, Phys. Rev. Lett. 99, 126601 (2007).

[16] J. P. Miller, E. de Rafael, and B. L. Roberts, Muon $(g-2)$ : Experiment and theory, Rep. Prog. Phys. 70, R03 (2007).

[17] R. J. Adler, The three-fold theoretical basis of the gravity probe B gyro precession calculation, Classical Quantum Gravity 32, 224002 (2015).

[18] I. B. Khriplovich and A. A. Pomeransky, Equations of motion of a spinning relativistic particle in external fields, Exp. Theor. Phys. 86, 839 (1998).

[19] C. M. Will, The confrontation between general relativity and experiment, Living Rev. Relativity 17, 4 (2014).

[20] S. Babak, J. Gair, A. Sesana, E. Barausse, C. F. Sopuerta, C. P. L. Berry, E. Berti, P. Amaro-Seoane, A. Petiteau, and A. Klein, Science with the space-based interferometer LISA. V. Extreme mass-ratio inspirals, Phys. Rev. D 95, 103012 (2017).
[21] A. B. Balakin and V. A. Popov, Spin-axion coupling, Phys. Rev. D 92, 105025 (2015).

[22] G. Franciolini, A. Kehagias, and A. Riotto, Imprints of spinning particles on primordial cosmological perturbations, J. Cosmol. Astropart. Phys. 02 (2018) 023.

[23] A. A. Kirillov, Elements of the Theory of Group Representations (Springer-Verlag, Berlin, 1976).

[24] B. Kostant, Quantization and unitary representations, in Lectures in Modern Analysis and Applications III, Vol. 170, edited by C. T. Taam (Springer, Berlin, Heidelberg, 1970), pp. 87-208.

[25] J. M. Souriau, Structure of Dynamical Systems: A Symplectic View of Physics (Springer Science \& Business Media, New York, 2012), Vol. 149.

[26] S. L. Lyakhovich and D. S. Kaparulin, Worldsheets of spinning particles, Phys. Rev. D 96, 105014 (2017).

[27] D. S. Kaparulin, S. L. Lyakhovich, and I. A. Retuntsev, Worldsheet of a massive spinning particle in $d=4$ Minkowski space, Russ. Phys. J. 61, 2145 (2019).

[28] D. S. Kaparulin, S. L. Lyakhovich, and I. A. Retuntsev, Variational principle for cylindrical curves and dynamics of massive spinning particles in $D=3$ Minkowski space, AIP Conf. Proc. 2163, 090012 (2019).

[29] D. S. Kaparulin and I. A. Retuntsev, On the worldsheet of anyon in the external electromagnetic field, arXiv:2011 $.06386 \mathrm{v} 2$.

[30] A. Nersessian and E. Ramos, Massive spinning particles and the geometry of null curves, Phys. Lett. B 445, 123 (1998).

[31] A. Nersessian and E. Ramos, A geometrical particle model for anyonss, Mod. Phys. Lett. A 14, 2033 (1999).

[32] A. Nersessian, R. Manvelyan, and H. J. W. Muller-Kirsten, Particle with torsion on 3-D null curves, Nucl. Phys. B, Proc. Suppl. 88, 381 (2000).

[33] C. Duval, M. Elbistan, P. A. Horvathy, and P.-M. Zhang, Wigner-Souriau translations and Lorentz symmetry of chiral fermions, Phys. Lett. B 742, 322 (2015).

[34] C. Duval and P. A. Horvathy, Chiral fermions as classical massless spinning particles, Phys. Rev. D 91, 045013 (2015).

[35] Yu. Aminov, Differential Geometry and Topology of Curves (CRC Press, New York, 2000).

[36] L.S. Ko, On the decomposition of derivations and skewderivations on differential forms of degree $k=0$. A necessary and sufficient condition for a curve to lie on a circular cylinder, M.S. thesis, University of Hong Kong, 1966.

[37] D. Keren, E. Rivlin, I. Shimshoni, and I. Weiss, Reconizing $3 d$ objects using tactile sensing and curve invariants, J. Math. Imaging and Vision 12, 5 (2000).

[38] E. L. Starostin and G. H. M. van der Heijden, Characterization of cylindrical curves, Monatshefte fur Mathematik 176, 481 (2015).

[39] J. Walrave, Curves and surfaces in Minkowski space, Ph.D. thesis, Katholieke Universiteit Leuven, 1995.

[40] M. Mathisson, Republication of: New mechanics of material systems, Gen. Relativ. Gravit. 42, 1011 (2010).

[41] A. Papapetrou, Spinning test-particles in general relativity, Proc. R. Soc. A 209, 248 (1951). 